\title{
Graphene-templated approach to ultrathin silica nanosheets
}

\author{
KAN LanYan, ZHENG BingNa \& GAO Chao* \\ Key Laboratory of Macromolecular Synthesis and Functionalization (Ministry of Education), Department of Polymer Science and Engineering, \\ Zhejiang University, Hangzhou 310027, China
}

Received November 2, 2011; accepted March 30, 2012; published online June 15, 2012

\begin{abstract}
Graphene, a perfect two-dimensional (2D) nanostructure, is an ideal template for 2D material design. We developed a graphenetemplated method to synthesize 2D silica nanosheets through the crosslinking of poly(3-methacryloxypropyl trimethoxysilane)grafted graphene oxide (GO-g-PMPS), followed by pyrolysis at $700^{\circ} \mathrm{C}$ for $10 \mathrm{~h}$.
\end{abstract}

graphene oxide (GO), poly(3-methacryloxypropyl trimethoxysilane) (PMPS), 2D silica nanosheets

Citation: $\quad$ Kan L Y, Zheng B N, Gao C. Graphene-templated approach to ultrathin silica nanosheets. Chin Sci Bull, 2012, 57: 3026-3029, doi: 10.1007/s11434$012-5252-6$

Two-dimensional (2D) nanostructures have attracted increasing interests since the rise of graphene in $2004[1,2]$, and a number of $2 \mathrm{D}$ nanomaterials such as $\mathrm{BN}, \mathrm{MoS}_{2}$ and $\mathrm{Bi}_{2} \mathrm{Te}_{3}$ [3-5] have been obtained by either exfoliation of natural mineral or chemical method. As a perfect 2D model, graphene or chemically modified graphene could be used as template due to its large-scale availability to fabricate other 2D nanomaterials [6]. Despite the big progress made in the field of 2D nanomaterials, 2D silica nanosheets have rarely been reported. Silica nanostructures have been widely researched in recent years for their unique size-dependent properties which lead to potential utilization in both fundamental science and industrial research [7-9]. Consequently, 1D silica nanostructures (e.g. nanowires [9], nanotubes $[10,11]$ and nanorods [12]) as well as 3D silica nanostructures (e.g. nanoparticles [13,14] and nanocubes [15]) have been widely investigated. Recently, graphene-supported large area silica nanosheets have been reported by our group [16]. Wang et al. [17] and Lee et al. [18] have also reported the graphene-templated methods of mesoporous silica fabrication [19]. However, simple and reliable preparation of 2D silica nanosheets still remains a challenge to be solved.

Here, we report a graphene oxide (GO)-templated methodology to synthesize silica nanosheets. Three steps are included in this method, as shown in Scheme 1: (1) sili-

*Corresponding author (email: chaogao@zju.edu.cn) con-contained vinyl polymer grafting on GO, (2) chemical crosslinking of the polymer layers on GO sheets, (3) pyrolysis at $700^{\circ} \mathrm{C}$ in air.

\section{Experimental}

Poly(3-methacryloxypropyl trimethoxysilane) (PMPS) was covalently grafted on GO sheets by in situ free radical polymerization approach presented previously by our group [20], affording PMPS-grafted GO brushes (Sample 1). The resulting Sample 1 were crosslinked in aqueous ammonia solution for $24 \mathrm{~h}$ [21]. Then, the crosslinked GO-g-PMPS (Sample 2) was collected and dispersed in water and freezedried. Finally, the dried powder of Samples 1 and 2 were both treated with pyrolysis at $700^{\circ} \mathrm{C}$ in air for $10 \mathrm{~h}$. After the decomposition of graphene template and other carbon units, Sample 1 gave rise to small pieces of silica clusters (Sample 3) and Sample 2 gave rise to silica nanosheets (Sample 4). The resultant products were characterized by thermal gravimetric analysis (TGA), atomic force microscopy (AFM), scanning electron microscope (SEM), transmission electron microscope (TEM), Raman spectroscopy and so on.

\section{Results and discussion}

$\mathrm{AFM}$ is considered to be the most effective equipment for 

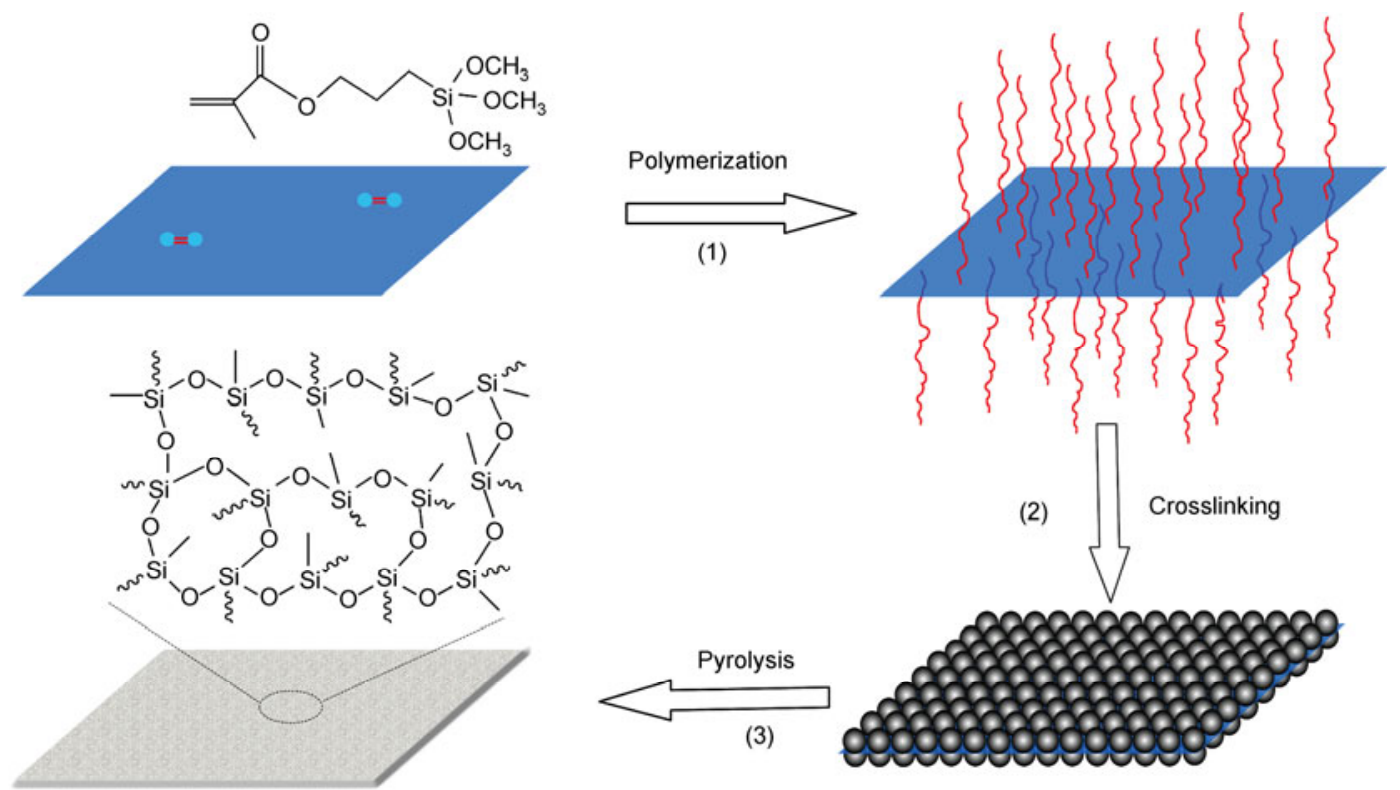

Scheme 1 (Color online) The synthesis protocol for two-dimensional silica nanosheets. Conditions: (1) In DMF, at $65^{\circ} \mathrm{C}$ for $24-36 \mathrm{~h}$; (2) $25 \%$ aqueous ammonia solution at room temperature for $24 \mathrm{~h}$; (3) pyrolysis in air at $700^{\circ} \mathrm{C}$ for $10 \mathrm{~h}$.

the visualization of graphene and its hybrid structures [5,22-25]. Figure 1(a) shows the AFM images of Sample 1 at different synthesis stages. Various protuberances, tufts of polymeric hairs, are evenly distributed on GO sheet after PMPS grafting. The height of Sample 1 reached to $5 \mathrm{~nm}$, much higher than pristine GO sheet $(\sim 0.8 \mathrm{~nm})$ [26], indicating the successful grafting of PMPS on GO sheet. Uniform and continuous sandwich-like layer without obvious cleavages was obtained after the crosslinking of grafted PMPS (Sample 2, Figure 1(b)). Obviously, independent polymer protuberances crosslink with each other and develop into a continuous network through the chemical crosslinking. A pyrolysis process at $700^{\circ} \mathrm{C}$ in air for $10 \mathrm{~h}$ was employed to generate template-free $2 \mathrm{D}$ nanosheets. The sample without crosslinking decomposed into small pieces after pyrolysis (Sample 3, Figure 1(c)), while the crosslinked sample treated with pyrolysis still maintains a continuous form revealing the integrity of the silica nanosheets (Sample 4, Figure 1(d)). This is due to the continuous network forms from chemical crosslinking of grafted PMPS, which can serve as the backbone of silica nanosheets after the pyrolysis. Without the backbone and GO template, Sample 3 cannot maintain the morphology and naturally decomposed into pieces. From FT-IR spectra, intensive peaks at 660 and $1090 \mathrm{~cm}^{-1}$ which ascribe to stretching vibration of $\mathrm{Si}-\mathrm{C}$ and asymmetric vibration of $\mathrm{Si}-\mathrm{O}-\mathrm{Si}$ were shown in Samples 1 and 2 while no such peaks can be detected for pristine GO. This is another evidence of successful grafting of PMPS to GO.

Figure 2(a) shows the TGA curves of GO and Sample 1, and the weight loss of Sample 1 is obviously higher than GO which is another evidence of the successful grafting of PMPS on GO sheet [27]. Raman spectroscopy is useful in
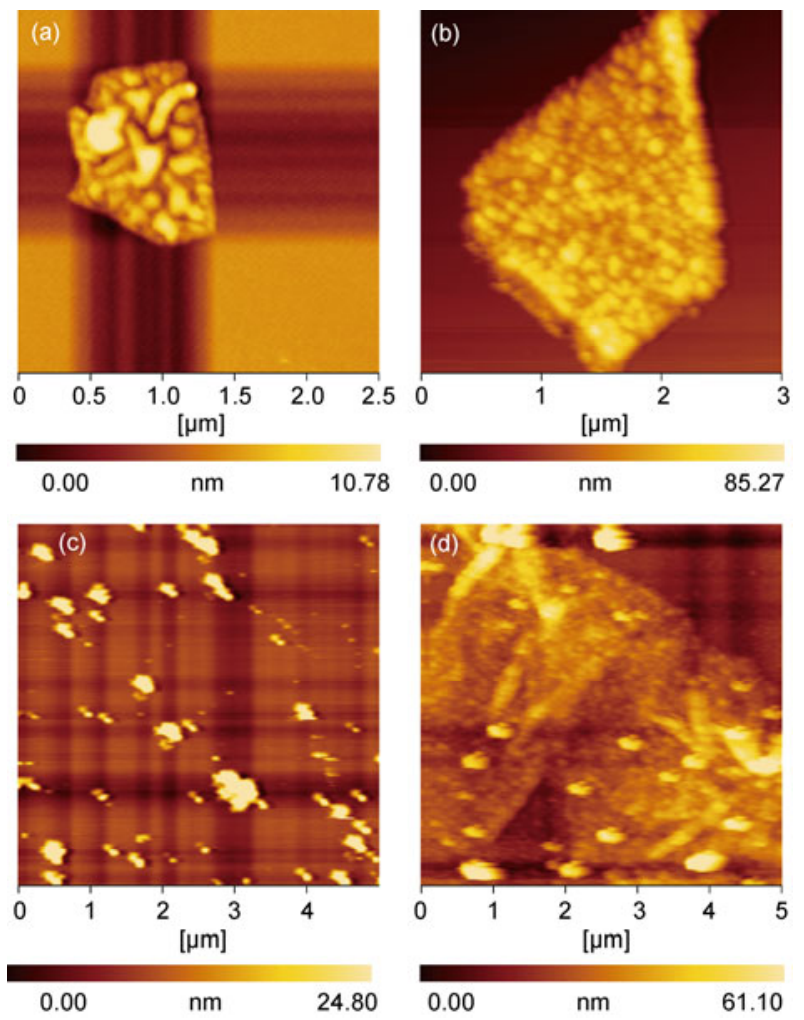

Figure 1 (Color online) AFM images of GO-g-PMPS after different treatments. (a) As-prepared Sample 1; (b) Sample 2; (c) Sample 3; (d) Sample 4.

probing $\mathrm{sp}^{3}$ and $\mathrm{sp}^{2}$ hybridized carbon atoms which was employed to trace the transformation from Sample 1 to Sample 4. In Figure 2(b), the two intensive peaks of GO at 1333 and $1592 \mathrm{~cm}^{-1}$ correspond to $\mathrm{D}$ band representing 

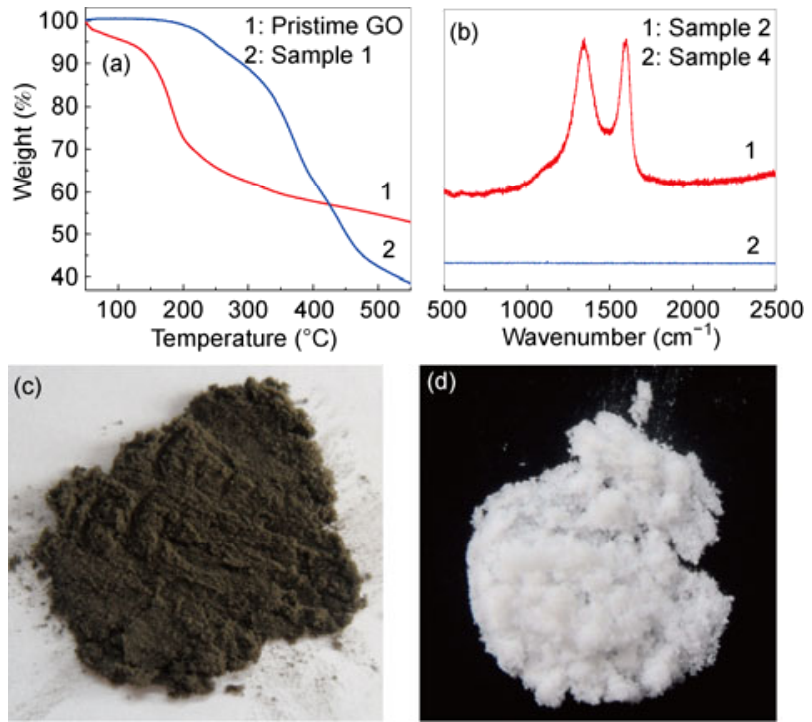

Figure 2 (Color online) (a) TGA curves of pristine GO and Sample 1; (b) Raman spectra of Sample 2 and Sample 4; powder of Sample 2 (c) and Sample 4 (d).

disordered $\mathrm{sp}^{3}$ carbon structure and $\mathrm{G}$ band of $\mathrm{sp}^{2}$ ordered crystalline graphite-like structures, respectively [5,28-31]. On the contrary, Sample 4 showed no apparent peak in the whole testing range. The vanishment of $\mathrm{D}$ and $\mathrm{G}$ bands is a strong evidence that the $\mathrm{sp}^{3}$ and $\mathrm{sp}^{2}$ carbon structures of GO decomposed totally after the pyrolysis process. Figure 2(c) and (d) exhibit the color change from black gray (Figure 2(c)) to pure white (Figure 2(d)) after pyrolysis, giving the direct visualization that the carbon in GO and the grafted carbon sources were completely burned out. This further indicated that pure 2D silica nanosheets were obtained through our method.

Representative TEM images are displayed in Figure 3 for further observation of the sample morphology. Comparing with smooth pristine GO (Figure 3(a)), various of protuberances are uniformly distributed on the nanosheet of Sample 1 (Figure 3(b)), which is in accordance with the result of AFM. In Figure 3(c), several evenly dispersed nanosheets of Sample 2 are observed and each sheet is attached with tufts of polymer protuberances. The corresponding magnified image (Figure 3(d)) shows that it is decorated with a thin and soft polymer layer resulting from the crosslinking of the grafted PMPS. Sample 4 in Figure 3(e) exhibit that several monolayer silica nanosheets which still maintain intact sheet morphology similar to the GO template are obtained. It indicates that the morphology of prepared silica nanosheets can be controlled through design of the templates. From the magnified image of silica nanosheet in Figure 3(f), we can find that rigid rough surface different from the soft polymer layer in Figure 3(d) are dispersed on its surface.

To investigate the original form of Sample 1 and silica nanosheets dispersed in water, freeze drying was adopted after crosslinking. As shown in Figure 2(d), the final sample

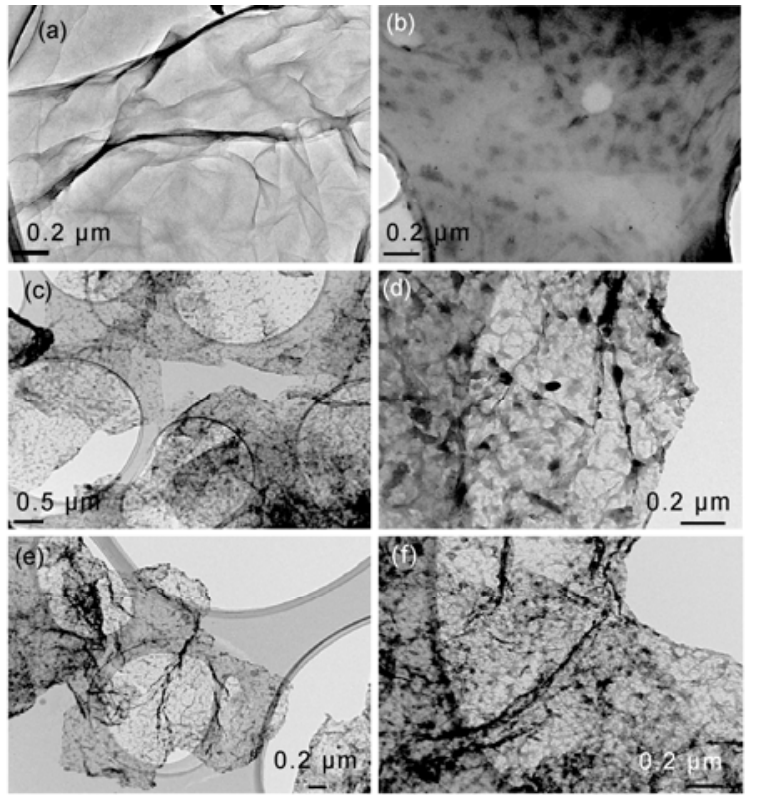

Figure 3 TEM images of GO (a), Sample 1 (b), Sample 2 (c), (d) and Sample 4 (e), (f).

is loose white powder. It is worth mentioning that Sample 1 is insoluble in water before crosslinking, whereas it can be dispersible in water after crosslinking. The final sample of silica nanosheets (Sample 4) is also dispersible in water, which lays the foundation of further functionalization and utilization.

Figure 4(a) shows the morphology of Sample 1, and it can be found that dispersed nanosheets are equipped with a shaggy surface differing from the smooth surface of pristine GO nanosheets [32]. One sheet with visible serried clusters densely coated on the flat surface is displayed in Figure 4(b). As for Sample 2, scattered nanosheets are still visible and each surface is developed into a uniform layer (Figure 4(d)) rather than a coating of serried clusters in Figure 4(b). From Figure 4(e), Sample 2 was turned into silica nanosheets (Sample 4) and could be dispersed in water with sheet morphology. Interestingly, almost all the nanosheets were continuous and intact except one hole indicating that grafted PMPS were not entirely coated on such sheet template in Figure 4(f). Consequently, the results obtained from TEM and SEM are consistent with each other, showing the flake morphology of the prepared 2D silica nanosheets through graphene oxide-templated method.

\section{Conclusion}

In summary, we developed a GO-templated method for facile, large-scale and shape controlled preparation of 2D silica nanosheets. By free radical polymerization, the siliconcontained vinyl polymer was effectively grafted onto GO nanosheets. Subsequently, water-dispersible 2D silica nano- 

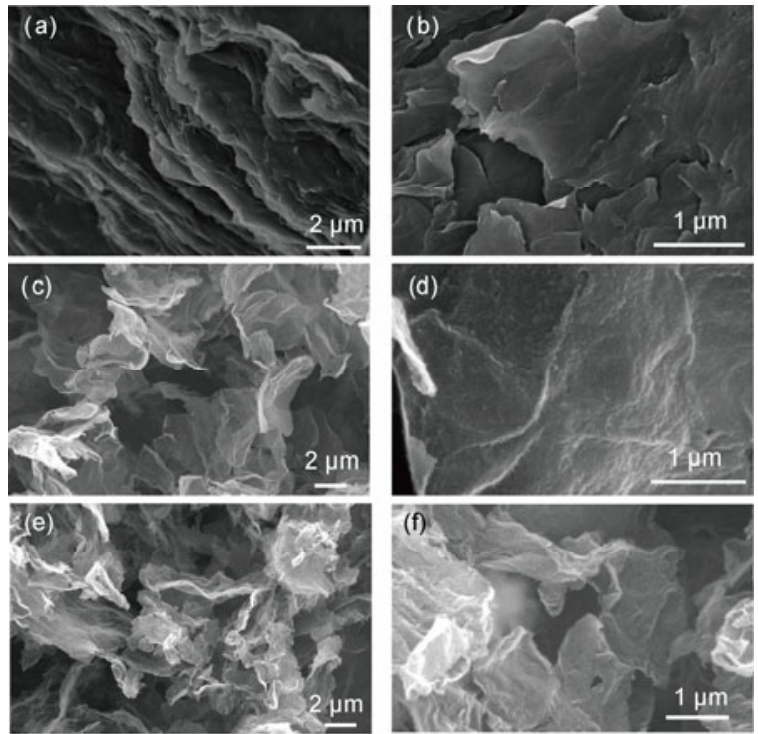

Figure 4 SEM images of GO-g-PMPS in different forms. (a), (b) Sample 1; (c), (d) Sample 2; (e), (f) Sample 4.

sheets were obtained after decomposition of GO template by treatments of crosslinking and pyrolysis. Owing to the large size and unique flat morphology, 2D silica nanosheets hold great promise in many applications.

This work was supported by the National Natural Science Foundation of China (51173162 and 20974093), Research Fund for the Doctoral Program of Higher Education of China (20100101110049), the Fundamental Research Funds for the Central Universities (2011QNA4029), Qianjiang Talent Foundation of Zhejiang Province (2010R10021), and Zhejiang Provincial Natural Science Foundation of China (R4110175).

1 Novoselov K S, Geim A K, Morozov S V, et al. Two-dimensional gas of massless Dirac fermions in graphene. Nature, 2004, 438: 197-200

2 Huang Y, Chen Y S. Functionalization of graphene and their applications (in Chinese). Sci China Ser B-Chem, 2009, 39: 887-896

3 Novoselov K S, Jiang D, Booth T J, et al. Two-dimensional atomic crystals. Proc Natl Acad Sci USA, 2005, 102: 10451-10453

4 Coleman J N, Lotya M, O'Neill A, et al. Two-dimensional nanosheets produced by liquid exfoliation of layered materials. Science, 2011, 331: 568-571

5 Rao C N R, Sood A K, Subrahmanyam K S, et al. Graphene: The new two-dimensional nanomaterial. Adv Mater, 2009, 48: 7752-7777

6 Huang X, Li S Z, Huang Y Z, et al. Synthesis of hexagonal close-packed gold nanostructures. Nat Commun, 2010, 2: 292

7 Lin J, Huang Y, Zhang J, et al. Characterization and photoluminescence properties of $\mathrm{Tb}$-Doped $\mathrm{SiO}_{2}$ nanowires as a novel greenemitting phosphor. Chem Mater, 2007, 19: 2585-2588

8 Petrovic Z S, Javni I, Waddon A, et al. Structure and properties of polyurethane-silica nanocomposites. J Appl Polym Sci, 2000, 76: $133-151$

9 Wang Z L, Dai Z R, Gao R P, et al. Side-by-side silicon carbidesilica biaxial nanowires: Synthesis, structure, and mechanical proper- ties. Appl Phys Lett, 2000, 77: 3349-3351

10 Kovtyukhova N I, Mallouk T E, Mayer T S, et al. Templated surface sol-gel synthesis of $\mathrm{SiO}_{2}$ nanotubes and $\mathrm{SiO}_{2}$-insulated metal nanowires. Adv Mater, 2003, 15: 780-785

11 Fan R, Wu Y, Li D, et al. Fabrication of silica nanotube arrays from vertical silicon nanowire templates. J Am Chem Soc, 2003, 125: 5254-5255

12 Zhu G, Zou X P, Cheng J, et al. Preparation of silica nano-rods by CVD. Adv Mater Res, 2008, 47-50: 359-362

13 Jin Y, Lohstreter S, Pierce D T, et al. Silica nanoparticles with continuously tunable sizes: Synthesis and size effects on cellular contrast imaging. Chem Mater, 2008, 20: 4411-4419

$14 \mathrm{Mu} \mathrm{X}, \mathrm{Wu} \mathrm{C} \mathrm{L,} \mathrm{Lai} \mathrm{J} \mathrm{P,} \mathrm{et} \mathrm{al.} \mathrm{A} \mathrm{facile} \mathrm{and} \mathrm{general} \mathrm{approach} \mathrm{for} \mathrm{the}$ synthesis of fluorescent silica nanoparticles doped with inert dyes. Chin Sci Bull, 2011, 56: 3242-3246

15 Yu K F, Guo Y P, Ding X F, et al. Synthesis of silica nanocubes by sol-gel method. Mater Lett, 2005, 59: 4013-4015

16 Kou L, Gao C. Making silica nanoparticle-covered graphene oxide nanohybrids as general building blocks for large-area superhydrophilic coatings. Nanoscale, 2011, 3: 519-528

17 Wang Z M, Wang W D, Coombs N, et al. Graphene oxide-periodic mesoporous silica sandwich nanocomposites with vertically oriented channels. ACS Nano, 2010, 4: 7437-7450

18 Lee Y F, Chang K H, Hu C C, et al. Graphene: A novel template for controlling the microstructures of mesoporous silica. J Mater Chem, 2011, 21: 14008-14012

19 Zhang X A, Jia H H, Wang X F, et al. Biosensors based on acetylcholinesterase immobilized on mesoporous silica thin films. Chin Sci Bull, 2009, 54: 3023-3028

20 Kan L Y, Xu Z, Gao C. General avenue to individually dispersed graphene oxide-based two-dimensional molecular brushes by free radical polymerization. Macromolecules, 2011, 44: 444-452

21 Yuan J Y, Xu Y Y, Walther A, et al. Water-soluble organo-silica hybrid nanowires. Nat Mater, 2008, 7: 718-722

$22 \mathrm{He} \mathrm{H} \mathrm{K,} \mathrm{Gao} \mathrm{C.} \mathrm{Supraparamagnetic,} \mathrm{conductive,} \mathrm{and} \mathrm{processable}$ multifunctional graphene nanosheets coated with high-density $\mathrm{Fe}_{3} \mathrm{O}_{4}$ nanoparticles. ACS Appl Mat Interfaces, 2010, 2: 3201-3210

23 He H K, Gao C. General approach to individually dispersed, highly soluble, and conductive graphene nanosheets functionalized by nitrene chemistry. Chem Mater, 2010, 22: 5054-5064

$24 \mathrm{Xu} \mathrm{Z}$, Gao C. Aqueous liquid crystals of graphene oxide. ACS Nano, 2011, 5: 2908-2915

25 Bai H, Li C, Shi G Q. Functional composite materials based on chemical converted graphene. Adv Mater, 2011, 23: 1089-1115

26 Stankovich S, Piner R D, Chen X Q, et al. Stable aqueous dispersions graphitic nanoplatelets via the reduction of exfoliated graphite oxide in the presence of poly(sodium 4-styrenesulfonate). J Mater Chem, 2006, 16: 155-158

$27 \mathrm{Xu} \mathrm{Z,} \mathrm{Gao} \mathrm{C.} \mathrm{In} \mathrm{situ} \mathrm{polymerization} \mathrm{approach} \mathrm{to} \mathrm{graphene-reinforced}$ nylon-6 composites. Macromolecules, 2010, 43: 6716-6723

28 Haddon R C. Chemistry of single-walled carbon nanotubes. Acc Chem Res, 2002, 35: 1105-1113

29 He H K, Gao C. A general strategy for the preparation of carbon nanotubes and graphene oxide decorated with $\mathrm{PdO}$ nanoparticles in water. Molecules, 2010, 15: 4679-4694

$30 \mathrm{He} \mathrm{H} \mathrm{K}$, Gao C. Graphene nanosheets decorated with Pd, Pt, Au, and Ag nanoparticles: Synthesis, characterization, and catalysis applications. Sci China Chem, 2011, 54: 397-404

$31 \mathrm{Xu} \mathrm{Z,} \mathrm{Gao} \mathrm{C.} \mathrm{Graphene} \mathrm{chiral} \mathrm{liquid} \mathrm{crystal} \mathrm{and} \mathrm{macroscopic} \mathrm{assem-}$ bled fibres. Nat Commun, 2011, 2: 571

32 Wan X J, Long G K, Chen Y S. Graphene-A promising material for organic photovoltaic cells. Adv Mater, 2011, 23: 5342-5358

Open Access This article is distributed under the terms of the Creative Commons Attribution License which permits any use, distribution, and reproduction in any medium, provided the original author(s) and source are credited. 